\title{
The BioBreeding rat diabetes model is infected with Ljungan virus
}

\author{
Bo Niklasson • T. Hultman • R. Kallies • M. Niedrig • \\ R. Nilsson • P.-O. Berggren • L. Juntti-Berggren • \\ S. Efendic • A. Lernmark • W. Klitz
}

Received: 27 February 2007 / Accepted: 28 February 2007 / Published online: 4 April 2007

(C) Springer-Verlag 2007

To the Editor: A useful tool for understanding the pathogenesis of human diseases has been the use of animal models. The BioBreeding (BB) rat has been a useful source of knowledge for type 1 diabetes, with over 1,400 publications recorded in PubMed. We now present evidence that this animal model is infected with a picornavirus.

We previously reported the covariation of the incidence of type 1 diabetes and the 3-4 year population cycles of the

\author{
B. Niklasson $(\bowtie) \cdot$ T. Hultman \\ Apodemus, \\ Grevgatan 38, \\ 11453 Stockholm, Sweden \\ e-mail: Bo.Niklasson@Apodemus.se
}

\section{B. Niklasson}

Department of Medical Cell Biology, Uppsala University,

Uppsala, Sweden

R. Kallies $\cdot$ M. Niedrig

Robert Koch Institute,

Berlin, Germany

R. Nilsson · P.-O. Berggren • L. Juntti-Berggren $\cdot$ S. Efendic The Rolf Luft Research Centre for Diabetes and Endocrinology, Karolinska Institute,

Stockholm, Sweden

\section{A. Lernmark}

R. H. Williams Laboratory, Department of Medicine, University of Washington,

Seattle, WA, USA

W. Klitz

School of Public Health, University of California, Berkeley, CA, USA

W. Klitz

Public Health Institute,

Oakland, CA, USA bank vole (Clethrionomys glareolus) in Sweden [1]. This led us to hypothesise that the bank vole is the reservoir and/ or vector of an infectious agent causing this disease in a predisposed population of humans. The hypothesis was supported by the observation that wild-trapped bank voles developed symptoms of diabetes in captivity [2]. A novel picornavirus, Ljungan virus (LV), has been isolated from diabetic bank voles in Sweden. Furthermore, viral antigen and picorna viral-like particles were detected by immunohistochemistry (IHC) and electron microscopy in affected pancreatic beta cells $[2,3]$. Analysis of new and stored samples have shown that LV is also present in wild voles in Denmark and the USA [4]. In addition, we have recently reported that clinical diabetes occurs in several other species of wild voles and lemmings in northern Sweden, extending the host range of LV, suggesting that this virus may cause significant morbidity among wild small rodents in general [5]. Outbred CD-1 laboratory mice develop glucose intolerance at 10-15 weeks of age following exposure to LV in utero or shortly after birth [6]. The wide host range of $\mathrm{LV}$, the association with diabetes in wild rodents, and the finding that $\mathrm{LV}$ induces glucose intolerance under controlled conditions in laboratory mice led us to investigate the $\mathrm{BB}$ rat.

All experimental procedures described below were approved by the animal ethics committee in Stockholm (N9/05) and in accordance with international guidelines (NIH publications no. 85-23, revised 1985). Formalin-fixed paraffin-embedded pancreas tissue was stained using IHC. We used LV VP1-specific polyclonal rabbit antisera and a mouse monoclonal antibody reactive to all known LV isolates but not to parecho- or cardiovirus. We also used three independent real-time RT-PCR assays to identify and quantify LV infection. One PCR assay used primers and two different minor-groove-binder probes designed to 


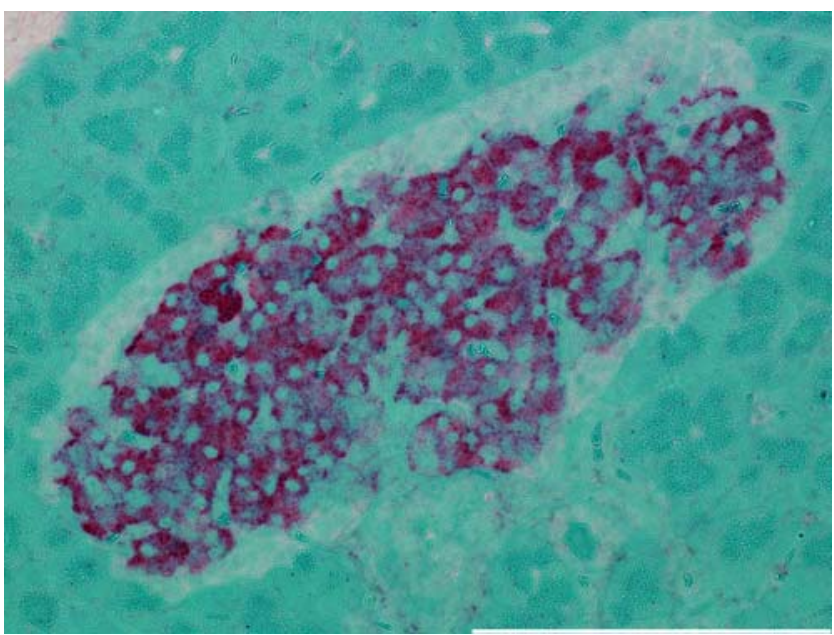

Fig. 1 Beta cell islet and adjacent acini from a non-diabetic BB rat pancreas at 40 days of age. The islet is stained with an LV-specific monoclonal antibody (red) and the background is stained with methyl green. Negative control normal mouse serum showed no LV-specific staining. The specific staining could be blocked with LV antigen but not with control antigen

amplify a $187 \mathrm{bp}$ PCR product from the $5^{\prime}$ untranslated region of the LV genome. In addition, we used primers designed to amplify a $103 \mathrm{bp}$ PCR product from the $5^{\prime}$ untranslated region and a $164 \mathrm{bp}$ product from the $2 \mathrm{~A}$ region of the LV genome, followed by a melting point analysis at the end of the amplification.

A total of 16 BB rats from a laboratory in Sweden (The Rolf Luft Research Centre for Diabetes and Endocrinology, Karolinska Institute, Stockholm) and 10 BB rats from the USA (R. H. Williams Laboratory, Department of Medicine, University of Washington, Seattle, WA, USA) all tested positive for LV by IHC. Positive staining was found in several organs, indicating a systemic infection. In the pancreas the staining was localised to the islets (Fig. 1). LV infection was confirmed in BB rats, from both continents, with positively immunostained sections by PCR. The highest RNA copy number from each individual, as measured by PCR, most often came from the brain tissue [7].

Additionally, ten Wistar and five Sprague-Dawley rats from Sweden (Scanbur, Sollentuna) also tested positive for LV by IHC staining, indicating that the virus may be found in several, possibly all, strains of rats. The presence of LV in samples from rats from Sweden, and the US suggests that LV may be characteristic of the strains and populations tested, rather than reflecting incidental infections at single locations. We present no data generated from BB rats directly proving that $\mathrm{LV}$ is involved in the development of the disease in these animals, but the evidence from native rodents and the causative role for LV in diabetes in a CD-1 mouse model show that LV may itself be a pathogenic agent of diabetes. Studies are under way to determine whether specific antiviral therapy can alter the clinical outcome of $\mathrm{BB}$ rats.

The demonstration that $\mathrm{BB}$ rats are infected with $\mathrm{LV}$ suggests that this animal model of spontaneous diabetes needs to be reconsidered. Additional work is needed to extend the findings of this study to the larger context of rodent susceptibility and carriage of LV, including the human commensal Rattus norvegicus and its laboratory strains. Regardless of the wider implications of this finding, it is clearly essential that a common pathogen with the potential to cause or interfere with disease development must be placed under exacting experimental control in an animal disease model.

Acknowledgements We are grateful for the excellent technical assistance of A. Kawecki. This work was supported in part by National Institutes of Health grant NIDDK064365 and the Barndiabetesfonden (Swedish Children's Diabetes Fund).

\section{References}

1. Niklasson B, Hornfeldt B, Lundman B (1998) Could myocarditis, insulin-dependent diabetes mellitus, and Guillain-Barré syndrome be caused by one or more infectious agents carried by rodents? Emerg Infect Dis 4:187-193

2. Niklasson B, Hornfeldt B, Nyholm E et al (2003) Type 1 diabetes in Swedish bank voles (Clethrionomys glareolus): signs of disease in both colonized and wild cyclic populations at peak density. Ann NY Acad Sci 1005:170-175

3. Niklasson B, Kinnunen L, Hornfeldt B et al (1999) A new picornavirus isolated from bank voles (Clethrionomys glareolus). Virology 255:86-93

4. Whitney E, Roz AP, Rayner GA (1970) Two viruses isolated from rodents (Clethrionomys gapperi and Microtus pennsvlvanicus) trapped in St. Lawrence County, New York. J Wildl Dis 6:48-55

5. Niklasson B, Nyholm E, Feinstein RE, Samsioe A, Hornfeldt B (2006) Diabetes and myocarditis in voles and lemmings at cyclic peak densities induced by Ljungan virus? Oecologia 150:1-7

6. Niklasson B, Samsioe A, Blixt M et al (2006) Prenatal viral exposure followed by adult stress produces glucose intolerance in a mouse model. Diabetologia 49:2192-2199

7. Donoso Mantke O, Kallies R, Niklasson B, Nitsche A, Niedrig M (2006) A new quantitative real-time reverse transcriptase PCR assay and melting curve analysis for detection and genotyping of Ljungan virus strains. J Virol Methods 141:71-77 